\title{
Whole grain consumption has a modest effect on the development of diabetes in the Goto-Kakisaki rat
}

\author{
Moonyeon Youn, A. Saari Csallany and Daniel D. Gallaher* \\ Department of Food Science and Nutrition, University of Minnesota, 1334 Eckles Avenue, St Paul, MN 55108, USA
}

(Received 15 October 2010 - Revised 5 April 2011 - Accepted 5 April 2011 - First published online 30 June 2011)

\begin{abstract}
Epidemiological evidence suggests that whole grain intake is associated with reduced risk of type 2 diabetes. However, studies of individual whole grains on the prevention of type 2 diabetes are lacking. The objective of the present study was to examine the effect of different whole grains on type 2 diabetes in an animal model of type 2 diabetes, the Goto-Kakisaki (GK) rat. GK rats were fed either a basal diet or a whole grain-containing diet for 5 months. Whole grain diets contained $65 \%$ whole grain flours of wheat, barley, oats or maize. After 2 months of feeding, fasting plasma glucose concentrations were lower in the wheat, barley and oats groups, compared with the basal group, whereas glycated $\mathrm{Hb}$ was significantly greater in the wheat group compared with other groups. Feeding of whole barley and maize increased plasma C-peptide concentrations compared with whole wheat at 2 months. There was a trend in the improvement of insulin resistance with a consumption of barley and oats diets at 2 months $(P=0.06)$ compared with the basal diet. Oxidative stress markers, urinary thiobarbituric acid-reactive substances and 8-isoprostane, did not improve with whole grain intake at 2 months. At 5 months, whole grain diets did not differ from the basal diet in glycaemic control, insulin secretion, oxidative stress and preservation of pancreatic $\beta$-cell mass. These results suggest that the consumption of whole grains may offer modest benefit early in the development of type 2 diabetes, but this benefit is lost with further development of the disease.
\end{abstract}

Key words: Whole grains: Diabetes: Glucose: Insulin sensitivity: $\beta$-Cells: Rats

The prevalence of type 2 diabetes has dramatically increased over the last several decades throughout the world ${ }^{(1)}$. This has resulted in considerable increases in morbidity and mortality related to type 2 diabetes, as well as a large economic cost for caring for those with the disease, creating an increasingly burdensome global health problem $^{(2)}$. Consequently, there is an urgent need for interventions that prevent or delay the development of type 2 diabetes and ameliorate its complications. Although there are a number of modifiable risk factors for type 2 diabetes, diet is clearly of paramount importance $^{(3)}$. However, which specific dietary components may be effective in reducing the incidence and progression of type 2 diabetes remains incompletely understood.

Several prospective cohort studies have shown a strong inverse relationship between the consumption of whole grain foods and the risk of developing type 2 diabetes ${ }^{(4-6)}$. There is also evidence that increased whole grain consumption improves blood glucose control and reduces insulin resistance and related diabetes risk factors, including oxidative stress $^{(7,8)}$. Although whole grain intake did not demonstrate a beneficial effect on insulin sensitivity in healthy subjects ${ }^{(9,10)}$,
Pereira et al. ${ }^{(8)}$ showed that insulin resistance was significantly improved with the consumption of whole grain foods in overweight hyperinsulinaemic subjects. Thus, increased consumption of whole grain foods may be a useful dietary approach to lowering the risk of developing diabetes.

The mechanism by which whole grains might convey such a benefit is not well understood. Possible mechanisms include a reduced postprandial plasma glucose response and a reduction in oxidative stress. Viscous dietary fibres have repeatedly been shown to lower the postprandial glucose response, an effect that may decrease the risk for diabetes ${ }^{(11)}$. Several studies have demonstrated that viscous fibre such as $\beta$-glucans, which are present in high concentrations in oats and barley, improves glucose control in type 2 diabetes ${ }^{(12,13)}$. Viscous fibre may lower postprandial glycaemia by delaying gastric empting $^{(14,15)}$ or by slowing glucose absorption in the small intestine ${ }^{(16)}$. Reducing oxidative stress by dietary antioxidants may also contribute to a reduction in the incidence of type 2 diabetes. Several prospective cohort studies suggest an inverse relationship between dietary antioxidant intake and the incidence of type 2 diabetes ${ }^{(17,18)}$. Whole grains,

Abbreviations: CCr, creatinine clearance; GHb, glycated Hb; GK, Goto-Kakisaki; HOMA, homoeostasis model assessment; TBARS, thiobarbituric acidreactive substances.

*Corresponding author: Dr D. D. Gallaher, fax +1612625 5272, email dgallahe@umn.edu 
particularly bran fractions, are concentrated sources of antioxidant compounds. Compared with their refined counterparts, whole grains have two- to three-fold greater antioxidant capacity ${ }^{(19)}$. In a clinical trial by Jang et al. ${ }^{(7)}$, the consumption of a whole grain diet for 16 weeks significantly reduced markers of oxidative stress, plasma malondialdehyde and urinary 8-isoprostane, in subjects with coronary artery disease. Consumption of a diet containing whole grains favourably altered markers of antioxidant defence ${ }^{(20)}$ and reduced the level of markers of lipid peroxidation ${ }^{(7)}$, suggesting a decrease in oxidative stress.

The objective of the present study was to evaluate the effect of the consumption of different whole grains on diabetic control and diabetic progression in an animal model of type 2 diabetes, the Goto-Kakisaki (GK) rat. GK rats exhibit moderate but stable hyperglycaemia, insulin resistance and impaired glucose tolerance, which appears at 3-4 weeks of age ${ }^{(21)}$. The whole grain flours used, wheat, barley, oats or maize, were chosen because they varied in the two dietary factors that relate to the possible mechanisms by which whole grains may reduce the risk of developing type 2 diabetes, viscous fibre (as $\beta$-glucans) and the antioxidant capacity, thus providing the possibility of elucidating the characteristic(s) of whole grain that may protect against type 2 diabetes. To this end, glucose control, insulin resistance, $\beta$-cell dysfunction and oxidative stress were measured at an early and later stage of diabetes in this well-established animal model of type 2 diabetes.

\section{Materials and methods}

\section{Animals and diets}

Male GK rats (7-8-weeks old) weighing 151-200g were purchased from Taconic Farms, Inc. (Hudson, NY, USA). The animals were individually housed in wire-bottomed stainless steel cages in an air-conditioned room $\left(22 \pm 2^{\circ} \mathrm{C}\right.$, $55 \pm \%$ relative humidity) with a $12 \mathrm{~h}$ light $-12 \mathrm{~h}$ dark cycle. All experimental procedures were approved by the University of Minnesota Committee on Animal Care.

Two factors, the content of $\beta$-glucan and the antioxidant capacity, were used to choose the appropriate species of whole grains for the present study to allow a $2 \times 2$ factorial design. The selected whole grains were wheat, barley, oats and maize. As shown in Table 1 , barley and oats had equivalent $\beta$-glucan content but barley had more than double the antioxidant activity. Maize had relatively high antioxidant activity with very little $\beta$-glucan. Wheat had both low

Table 1. $\beta$-Glucan concentration and antioxidant activity of whole grains

\begin{tabular}{lcc}
\hline Whole grain & $\beta$-Glucan content (\%) & Antioxidant activity* \\
\hline Barley & 3.9 & 2871 \\
Oats & 3.9 & 759 \\
Maize & 0.07 & 1277 \\
Wheat & 0.65 & 838 \\
\hline
\end{tabular}

* Antioxidant activity was expressed as $\mu \mathrm{mol}$ Trolox equivalents $/ 100 \mathrm{~g}$ sample. $\beta$-glucan content and antioxidant capacity. Barley and oats were classified as having a high level of $\beta$-glucan and wheat and maize were classified as having a low level of $\beta$-glucan. Barley and maize were classified as having a high level of antioxidant capacity and oats and wheat were classified as having a low level of antioxidant capacity.

Whole grain flours were obtained from a local milling company (Whole Grain Milling Company, Welcome, MN, USA) and stored at $-20^{\circ} \mathrm{C}$. A proximate analysis was conducted by a commercial laboratory (Medallion Laboratories, Minneapolis, MN, USA) to obtain the protein, carbohydrate, lipid, total dietary fibre and ash content of the whole grain flours. The $\beta$-glucan concentration and antioxidant activity of the flours are shown in Table 1 . The $\beta$-glucan content was determined using an enzymatic spectrophotometric method ( $\beta$-Glucan Assay Kit; Megazyme, Wicklow, Ireland). Antioxidant capacity was measured by the 2,2-diphenyl-1-picrylhydrazyl assay, as described by Miller et al. ${ }^{(22)}$. A modification of the American Institute of Nutrition 93G purified diet was used as the basal diet. Whole grain diets contained $65 \%$ of wheat, barley, oats or maize flour. To allow comparison with human diets, this quantity of flour provided $14.8 \mathrm{~g} \beta$-glucan $/ 10000 \mathrm{~kJ}$ for the barley and oat flour-based diets, $0.3 \mathrm{~g} \beta$-glucan $/ 10000 \mathrm{~kJ}$ for the maize flour-based diet, and $2 \cdot 2 \mathrm{~g} \beta$-glucan $/ 10000 \mathrm{~kJ}$ for the wheat flour-based diet. Based on a proximate analysis of the flours, the macronutrient content (carbohydrate, protein, fat and total dietary fibre) of the whole grain diets were closely matched to that of the basal diet. The composition of the diets is shown in Table 2 . The diets were prepared every month and stored at $-20^{\circ} \mathrm{C}$.

\section{Experimental design}

The animals were adapted to the basal diet for $3 \mathrm{~d}$. A total of ten rats were randomly assigned to either the basal diet or one of the four whole grain diets. Diets were fed ad libitum for 5 months with free access to water. Body weight and food intake were measured bi-weekly and monthly, respectively. After 2 months of feeding, blood was collected from the retro-orbital sinus after a $12 \mathrm{~h}$ fast. A $24 \mathrm{~h}$ fasting urine collection was made at 2 and 5 months of the feeding period, beginning after a $12 \mathrm{~h}$ fast to eliminate lipid oxidation products emanating from the diets. At the end of the dietary treatment, the rats fasted for $12 \mathrm{~h}$ and were anaesthetised with isoflurane and blood was collected by cardiac puncture into EDTA-containing syringes. The kidneys and epididymal fat pads were removed and weighed. The pancreas was removed, weighed and embedded in paraffin for immunohistochemistry.

\section{Biochemical analysis}

The percentage of glycated $\mathrm{Hb}$ (GHb) in whole blood was analysed by an affinity chromatographic method (Helena Glyco-Tek Affinity Column Method; Helena Laboratories, Beaumont, TX, USA). GHb represents all glycated fractions of $\mathrm{Hb}$, whereas $\mathrm{HbA}_{1 \mathrm{c}}$ represents $\mathrm{HbA}$ with glucose attached to the $\mathrm{NH}_{2}$-terminal valines of the $\beta$-chain. Although these two fractions are measured using different methods, clinically 
Table 2. Composition of the diets

\begin{tabular}{lccccc}
\hline $\begin{array}{l}\text { Diet ingredients } \\
\text { (g/kg diet) }\end{array}$ & Basal & Wheat & Barley & Oat & Maize \\
\hline Flour & 0 & 650 & 650 & 650 & 650 \\
Sucrose & 100 & $83 \cdot 3$ & $78 \cdot 1$ & $135 \cdot 3$ & $85 \cdot 9$ \\
Maize starch & $448 \cdot 2$ & 0 & 0 & 0 & 0 \\
Casein & 200 & $110 \cdot 95$ & $129 \cdot 15$ & $110 \cdot 3$ & $168 \cdot 67$ \\
Cellulose & 80 & $7 \cdot 85$ & $0 \cdot 05$ & $14 \cdot 35$ & $22 \cdot 15$ \\
Mineral mix & 35 & 35 & 35 & 35 & 35 \\
Vitamin mix $\dagger$ & 10 & 10 & 10 & 10 & 10 \\
L-Cystine & 3 & 3 & 3 & 3 & 3 \\
Choline & $2 \cdot 5$ & $2 \cdot 5$ & $2 \cdot 5$ & $2 \cdot 5$ & $2 \cdot 5$ \\
$\quad$ bitartrate & & & & & \\
Sunflower oil & 120 & $102 \cdot 39$ & $100 \cdot 83$ & $66 \cdot 38$ & $88 \cdot 67$ \\
Total weight & 1000 & 1000 & 1000 & 1000 & 1000 \\
Total & 55 & 55 & 55 & 55 & 55 \\
$\quad$ carbohydrate & & & & & \\
$\quad$ (\%) & & & & & \\
Total & 20 & 20 & 20 & 20 & 20 \\
$\quad$ protein (\%) & & & & & \\
Total fat (\%) & 12 & 12 & 12 & 12 & 12 \\
Total fibre (\%) & 8 & 8 & 8 & 8 & 8 \\
\hline * AIN-93G mineral mix. & & & & & \\
\hline AIN-93G vitamin mix. & & & & &
\end{tabular}

they are equivalent ${ }^{(23)}$. Fasting plasma glucose and NEFA concentrations were measured with an enzymatic colorimetric method using commercial kits (Autokit Glucose and HR Series NEFA-HR (2), respectively; WAKO Chemicals, Richmond, VA, USA). The concentrations of fasting plasma insulin and C-peptide were measured by RIA specific for rat insulin and C-peptide (Linco Research, St Charles, MO, USA). Urinary concentration of urinary thiobarbituric acid-reactive substances (TBARS) was measured as described by Mihara \& Uchiyama ${ }^{(24)}$ with minor modifications. Urine samples $(100 \mu \mathrm{l})$ were mixed with $300 \mu \mathrm{l}$ of $1 \%(\mathrm{v} / \mathrm{v})$ phosphoric acid and $200 \mu \mathrm{l}$ of $0.6 \%$ $(\mathrm{w} / \mathrm{v})$ thiobarbituric acid and heated at $100^{\circ} \mathrm{C}$ for $45 \mathrm{~min}$. After cooling, $100 \mu$ l of $n$-butanol was added to each tube and mixed vigorously to extract the colour reactant. The butanol layer was separated by centrifugation at $750 \boldsymbol{g}$ for $10 \mathrm{~min}$, collected, and the absorbance measured at $535 \mathrm{~nm}$. Malondialdehyde standards were prepared from malondialdehyde tetramethylacetal as described by Lee et $a l .{ }^{(25)}$ and treated as were the urine samples. Urinary 8 -iso- $\mathrm{PGF}_{2 \mathrm{a}}$ (8-isoprostane) concentrations were measured using an ELISA kit (Northwest Life Science Specialties; LLC, Vancouver, WA, USA). The concentration of creatinine in urine and plasma was determined by the Jaffe reaction using a commercially available kit (R\&D System, Minneapolis, MN, USA). Creatinine clearance (CCr) was calculated according to the following formula:

$$
\begin{aligned}
& \operatorname{CCr}(\mathrm{ml} / \mathrm{min})=(\text { creatinine in urine }(\mathrm{mg} / \mathrm{ml}) \\
& \quad \times \text { urine }(\mathrm{ml} / \mathrm{min})) /(\text { creatinine in plasma }(\mathrm{mg} / \mathrm{ml})) .
\end{aligned}
$$

\section{Calculation of insulin resistance}

The homoeostasis model assessment (HOMA), which is based on fasting plasma insulin and glucose concentrations, was used to estimate insulin resistance ${ }^{(26)}$. The formula is as follows:

$$
\begin{aligned}
\mathrm{HOMA}= & (\text { fasting insulin }(\mu \mathrm{U} / \mathrm{ml}) \\
& \times \text { fasting glucose }(\mathrm{mg} / \mathrm{dl})) / 405 .
\end{aligned}
$$

\section{Estimation of total $\beta$-cell mass}

The paraffin-embedded pancreas was sectioned into $7 \mu \mathrm{m} \mathrm{sec-}$ tions throughout its length. The section was counted when $25 \%$ of the section was occupied with pancreatic tissue. Every 30th to 35 th section was sampled on glass slides and was immunostained for insulin using guinea pig anti-insulin antibody (1:2000 dilution, a generous gift from Dr Robert L. Sorenson), followed by peroxidase-conjugated donkey anti-guinea pig IgG (1:500 dilution; Jackson Immunoreseach Laboratories, Inc., West Glove, PA, USA). The sections were developed with diaminobenzadine-containing nickel chloride, which yields a grey colour for $\beta$ cells (DAB Substrate Kit; Vector Laboratories, Inc., Burlingame, CA, USA). The sections were counterstained with Van Gieson's picric acid-fuchsin stain. The relative $\beta$-cell mass was estimated by the method of point counting on insulin antibody-stained sections of the pancreas $^{(27)}$. A microscope (Olympus BX40; Melville, NY, USA) connected to a digital camera (Olympus DP11-N) and monitor (Sony PVM-14N5U; Tokyo, Japan) were used to obtain digital images at $100 \times$ magnification. For each pancreas, 161-318 images were examined for the quantification of the $\beta$-cell volume. Adobe Photoshop 7.0 software was used to observe the images and superimpose counting grids over the same images. Using a point counting grid containing 192 fine points and one coarse point (encircled), the number of fine points hitting $\beta$-cells and the number of coarse points hitting pancreatic tissue was counted.

Total $\beta$-cell mass was calculated according to:

$$
\begin{aligned}
& \beta \text {-cell mass }(\mathrm{mg})=\text { pancreas }(\mathrm{mg}) \\
& \times \frac{\text { number of fine points hitting } \beta \text {-cells }}{\text { number of coarse points hitting pancreatic tissue } \times 193}
\end{aligned}
$$

\section{Statistical analysis}

The results are expressed as means with their standard errors. Diet group differences were analysed by one-way ANOVA, followed by Duncan's test to inspect individual differences among the diet groups. Groups fed the whole grain flours were also analysed by two-way ANOVA with two levels of antioxidant capacity (low and high) and $\beta$-glucan content (low and high) as the main effects. The results from the plasma and urinary assays obtained from the two time points were analysed by three-way ANOVA with factors of antioxidant capacity (low and high), $\beta$-glucan content (low and high) and time ( 2 and 5 months). Pearson correlation analysis was used to assess the correlations between the measurements. Values for $P<0.05$ were considered statistically significant. All statistical analyses were performed by using 
Statistical Analysis Systems statistical software package version 9.1 (SAS Institute, Cary, NC, USA).

\section{Results}

\section{Food intake, body and tissue weight}

Fig. 1 shows the changes in the body weight of GK rats fed either the basal or whole grain diets. Overall, the wheat group gained less weight than the other groups at each time point after 6 weeks of feeding. However, body weight gain averaged over the feeding trial did not differ among the diet groups. The rats fed the wheat diet consumed significantly less during the first month compared with rats in the basal group $(P<0.05)$. However, after 2 months, daily food consumption for the whole grain groups did not differ significantly from that of the basal group (data not shown).

Neither the absolute nor the relative weights of the pancreas or epididymal fat pads differed among the diet groups (Table 3). Although the absolute kidney weights did not differ among the groups, the animals fed the wheat and oats diets had a greater relative kidney weight (g/100g body weight) compared with the maize diet $(P<0.05)$. Two-way ANOVA revealed a significant main effect of antioxidant capacity on the relative kidney weight $(P=0.013)$, such that the groups fed diets with greater whole grain antioxidant capacity had lower relative kidney weight.

\section{Plasma glucose concentration}

At 2 months, the rats fed the wheat, barley and oats diets had significantly lower fasting plasma glucose concentrations compared with the basal group (Table $4 ; P<0 \cdot 05$ ). However, fasting plasma glucose concentrations in the oats group were significantly greater than all other groups at 5 months $(P<0.05)$. Fasting plasma glucose level significantly increased from 2 to 5 months $(P<0 \cdot 001)$. There was an interaction effect of time $\times \beta$-glucan on fasting plasma glucose $(P=0 \cdot 027)$, such

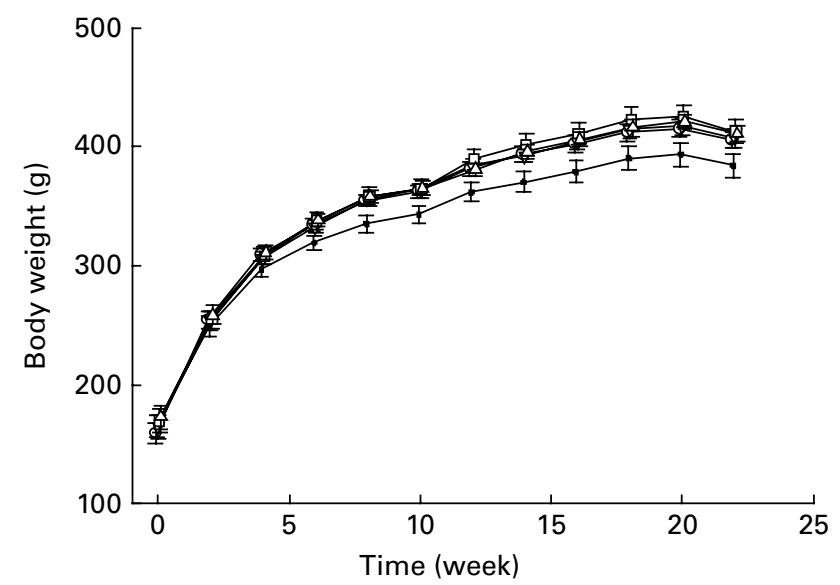

Fig. 1. Body weight changes of Goto-Kakisaki rats fed the basal (-○-), wheat $(-\bullet-)$, barley $(-\nabla-)$, oat $(-\square-)$ or maize-based $(-\Delta-)$ diets for 22 weeks. Values are means, with their standard errors represented by vertical bars, $n 9-10$.

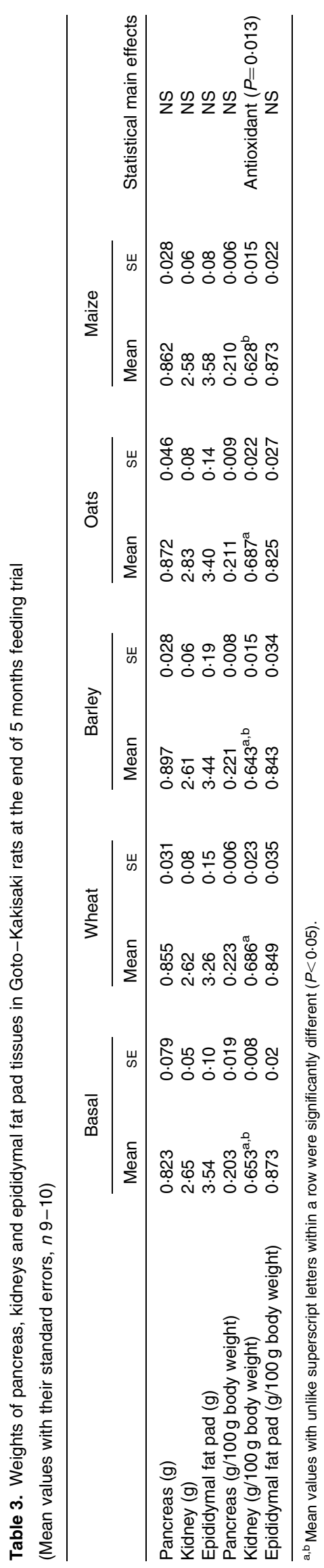


that animals fed diets with a high content of $\beta$-glucan had a lower fasting plasma glucose at 2 months but a greater concentration of plasma glucose at 5 months. The percentage of GHb was significantly greater in the wheat group at 2 and 5 months and the oats group at 5 months compared with the basal group (Table 4; $P<0 \cdot 05$ ). GHb also significantly increased from 2 to 5 months $(P<0 \cdot 001)$. Three-way ANOVA indicated that antioxidant capacity had a significant main effect on GHb $(P=0.045)$, such that the animals fed whole grains with a high antioxidant capacity had a lower GHb. In addition, GHb at 5 months was correlated with relative kidney weight $(r 0.546, P<0.0001)$.

\section{Plasma insulin and C-peptide concentration and insulin resistance}

Fasting plasma insulin levels did not differ among the groups and significantly decreased from 2 to 5 months in all diet groups (Table 4; $P=0$-004). Fasting plasma C-peptide concentrations were significantly greater in the barley and maize groups compared with the wheat group at 2 months $(P<0.05)$. Fasting plasma C-peptide levels decreased from 2 to 5 months in all groups except the wheat group (Table 4; $P<0.001)$. Three-way ANOVA indicated that antioxidant capacity had a significant main effect on C-peptide levels $(P=0.042)$, such that the diet groups with greater whole grain antioxidant capacity had greater fasting plasma $\mathrm{C}$-peptide concentrations. In addition, there was a significant interaction between time and $\beta$-glucan content $(P=0 \cdot 011)$, such that the diet groups with a high $\beta$-glucan content had a greater concentration of C-peptide at 2 months but a lesser concentration of plasma C-peptide at 5 months. Insulin resistance, determined by HOMA, was not significantly different among the groups at either 2 or 5 months (Table 4). However, there was a trend for a lower HOMA (less insulin resistance) with the barley and oats diets at 2 months $(P=0.06)$. Three-way ANOVA revealed a significant time effect on insulin resistance, such that resistance increased with time $(P=0.004)$. Fasting plasma glucose showed a significant inverse correlation to C-peptide concentration at 2 months $(r-0.33, P=0.021)$; however, at 5 months this correlation was no longer evident $(r-0.04, P=0 \cdot 79)$.

\section{Oxidative stress}

When the concentration of urinary TBARS was expressed as $\mu \mathrm{g} / 24 \mathrm{~h}$, the level of TBARS was significantly increased in the oats group at 2 months and the barley group at 5 months, compared with the other diet groups and the maize group, respectively (Table $5 ; P<0.05$ ). Three-way ANOVA indicated significant main effects of antioxidant capacity $(P=0.039)$ and $\beta$-glucan content $(P<0.001)$, such that the amount of urinary TBARS was decreased with the higher level of antioxidant capacity and the lower level of $\beta$-glucan.

The concentration of urinary 8-isoprostane, expressed as $\mathrm{ng} / 24 \mathrm{~h}$, is shown in Table 5 . At 2 months of diet treatment, the oats groups had a significantly greater concentration of 8-isoprostane compared with the barley and wheat groups 
$(P<0 \cdot 05)$. However, at 5 months, the maize diet had a significantly greater concentration of 8-isoprostane compared with the basal and wheat diets, and the oats group had a significantly greater level of 8 -isoprostane than the basal group $(P<0 \cdot 05)$. Three-way ANOVA revealed a significant interaction between antioxidant capacity and $\beta$-glucan content $(P<0 \cdot 001)$, such that the diet groups with the higher level of antioxidant capacity had lower concentrations of 8-isoprostane when the level of $\beta$-glucan content was low but when the level of $\beta$-glucan content was high, the concentration of 8 -isoprostane was increased with the high level of antioxidant capacity. In addition, at 5 months, urinary 8-isoprostane concentration $(\mathrm{ng} / 24 \mathrm{~h})$ was positively correlated with urinary TBARS $(\mu \mathrm{g} / 24 \mathrm{~h})(r 0.47, P<0.009)$.

\section{Plasma NEFA concentration and creatinine clearance}

Plasma NEFA concentrations did not differ among diet treatments at 5 months (Table 6). CCr, an indicator of glomerular hyperfiltration, was not significantly different among the diet groups at 5 months (Table 6). Two-way ANOVA indicated a significant main effect of $\beta$-glucan level on $\operatorname{CCr}(P=0 \cdot 016)$, such that the diet groups with a high $\beta$-glucan content had greater CCr.

\section{Relative volume of $\beta$-cells}

Fig. 2 shows representative pancreas sections stained for insulin with the point-counting grid overlaid. There were no significant differences in the mean total $\beta$-cell mass among the diet groups (data not shown).

\section{Discussion}

In order to identify which whole grains might be most effective in slowing the onset of type 2 diabetes, we investigated the effect of the consumption of four commonly consumed whole grains in diabetic control and progression in the GK rat. The GK rat is the most commonly used spontaneous animal model of type 2 diabetes mellitus, which was produced by repeated inbreeding of Wistar rats with mild glucose intolerance $^{(28)}$. GK rats exhibit hyperglycaemia, insulin resistance, impaired insulin secretion upon glucose stimulation and reduction of $\beta$-cell mass ${ }^{(21,29,30)}$. However, unlike most type 2 diabetes patients, the GK rat does not develop obesity and dyslipidaemia $^{(31,32)}$. Therefore, the GK rat appears to be an appropriate animal model to study the effect of whole grain intake on the pathogenesis of type 2 diabetes in the absence of such confounding factors. To our knowledge, this is the first study to examine and compare the effect of consumption of individual whole grains on diabetic control in an animal model of non-obese type 2 diabetes.

In the present study, we focused on two characteristics of whole grains that might act to delay the progression of diabetes. $\beta$-Glucans, a viscous fibre present in oats and barley, are well known for reducing the postprandial blood glucose response. High antioxidant capacity, such as found in barley and to a lesser extent in maize, may reduce the oxidative 
Table 6. The level of NEFA and creatinine clearance of Goto-Kakisaki rats fed either basal or whole grain diets at 5 months*

(Mean values with their standard errors, $n 9-10$ )

\begin{tabular}{|c|c|c|c|c|c|c|c|c|c|c|c|}
\hline & \multicolumn{2}{|c|}{ Basal } & \multicolumn{2}{|c|}{ Wheat } & \multicolumn{2}{|c|}{ Barley } & \multicolumn{2}{|c|}{ Oats } & \multicolumn{2}{|c|}{ Maize } & \multirow[b]{2}{*}{ Statistical main effect } \\
\hline & Mean & SE & Mean & SE & Mean & SE & Mean & SE & Mean & SE & \\
\hline Plasma NEFA (mmol/l) & 0.55 & 0.04 & 0.55 & 0.03 & 0.61 & 0.07 & 0.53 & 0.04 & 0.59 & 0.06 & NS \\
\hline Creatinine clearance $(\mathrm{ml} / \mathrm{min})$ & 0.94 & 0.07 & 1.02 & 0.06 & 1.06 & 0.07 & $1 \cdot 11$ & 0.08 & 0.83 & 0.04 & $\beta$-Glucan $(P=0.016)$ \\
\hline
\end{tabular}

* Data obtained from fasted rats.

stress associated with diabetes. These two factors, the content of $\beta$-glucan and the antioxidant capacity, were used to guide the choice of whole grains for the present study and as a way to explore the impact of whole grains on outcomes related to diabetes control and progression.

The results suggest that certain whole grains may have a modest beneficial effect early in the course of type 2 diabetes. Feeding of whole wheat, barley and oats for 2 months lowered fasting plasma glucose in GK rats relative to the grain-free basal diet. Further, we found evidence for an improvement in insulin resistance, as demonstrated by a strong trend for a lower HOMA, after the consumption of whole barley and oats for 2 months. These results are consistent with recent findings from clinical trials indicating that the consumption of whole grains may protect against type 2 diabetes by improving glucose control and reducing insulin resistance ${ }^{(7,8)}$. The whole grains used in those studies, however, were a mixture of grains, including wheat, oat, barley, rice and maize, thus making it impossible to determine the effect of individual whole grains on the development of type 2 diabetes. Nevertheless, in the present study, longer-term consumption of whole grains ( 5 months) did not demonstrate beneficial effects on glucose and insulin responses, insulin resistance, oxidative stress or on preserving $\beta$-cell mass.

Body weights among the dietary groups did not differ, with the exception of the wheat group, which had a significantly lower body weight than other groups during the last 4 months of feeding. A lower body weight might suggest a

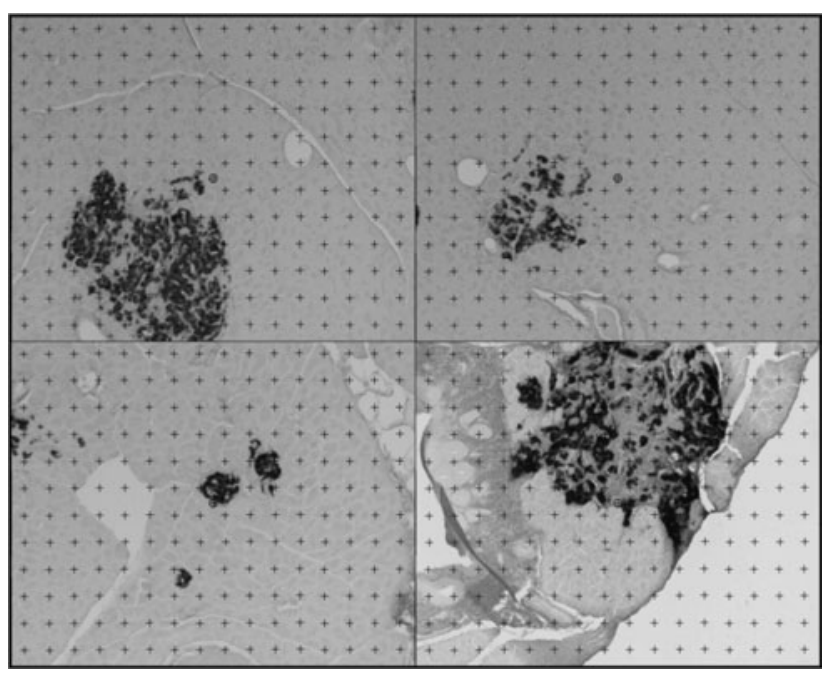

Fig. 2. Insulin immunostained pancreatic tissue sections from Goto-Kakisak rats with the point counting grid overlain. worsening of the diabetic state. However, measures of glucose control and insulin secretion did not differ between the wheat group and the other groups, suggesting that this was not the case. Thus, the reason for the lower body weight in the wheat group is unclear.

Increases in fasting plasma glucose and $\mathrm{GHb}$ level from month 2 to month 5 clearly indicated a progression of diabetes in the GK rats over the feeding period. After 2 months of feeding, the fasting plasma glucose concentration was significantly lower in the wheat, barley and oats groups compared with the basal group. In addition, fasting plasma glucose was decreased with the consumption of grains containing a high $\beta$-glucan content for 2 months whereas, surprisingly, the effect was reversed at the end of the feeding period. In type 2 diabetes, the reduction in postprandial glucose and insulin level by the consumption of $\beta$-glucan from oats and barley appears to be dose dependent ${ }^{(13,33)}$. Thus, it may be that the dietary $\beta$-glucan concentration in the present study was sufficient to improve glucose response at the 2 month time period, early in the progression of diabetes, but not sufficient to exert the effect at the end of the study when glucose control had greatly deteriorated. Surprisingly, GHb concentration was significantly greater at both time points in the wheat and oats groups compared with the basal group. It is unclear why the pattern of fasting plasma glucose and $\mathrm{GHb}$ concentrations did not parallel each other. GHb has been used as a measure of long-term blood glucose ${ }^{(34,35)}$. Formation of GHb is nonenzymatic and controlled by the concentrations of glucose and protein and the half-life of the glycated protein ${ }^{(35)}$. In addition, a high degree of oxidative stress also appears to increase glycation of $\mathrm{Hb}^{(36,37)}$, and reducing oxidative stress with vitamin $\mathrm{E}$ was found to reduce glycation of $\mathrm{Hb}$ in glucose-treated erythrocytes ${ }^{(38)}$. The antioxidant capacity of the grains had a significant main effect on GHb, suggesting that the consumption of grains with high antioxidant capacity may have reduced glycation of $\mathrm{Hb}$ relative to those with lower antioxidant capacity. Thus, early in the development of diabetes, whole grains appear to improve glucose control, but this improvement is not maintained as glucose control worsens with the progression of diabetes.

The decreased concentrations of plasma insulin and C-peptide from month 2 to month 5 indicate that insulin secretion was progressively impaired during the feeding period. Insulin and C-peptide are secreted in equimolar concentrations from pancreatic $\beta$-cells, but $C$-peptide has a longer half-life than insulin $^{(39)}$ and thus has been used as a marker for insulin secretion. In GK rats, impaired insulin secretion in response to glucose occurs in isolated islets and perfused pancreas 
from 8 weeks of age $\mathrm{e}^{(29,40,41)}$. Thus, the greater fasting plasma C-peptide concentrations in the barley and maize groups compared with that of the wheat group at 2 months suggest a slower development of diabetes in young GK rats with a consumption of the grains with higher antioxidant capacity. Yet, groups fed grains with high $\beta$-glucan content had the paradoxical effect of having greater C-peptide concentrations after 2 months of diet treatment, but lower concentrations after 5 months, as indicated by an interaction of the main effects of time and $\beta$-glucan. However, as C-peptide concentrations at 5 months in the whole grain groups did not differ from the basal group, it is likely that by 5 months the progression of diabetes overwhelmed the modest benefits provided by the whole grains at 2 months.

The HOMA values at 2 months showed a trend towards improvement in insulin resistance with the consumption of whole barley and whole oats diets $(P=0.06 v$. basal for both). It has been suggested that a correlation between insulin secretion and insulin resistance may be weakened or lost in overt diabetes when insulin secretion is impaired ${ }^{(42)}$. Therefore, at 5 months of diet treatment, the use of HOMA for calculating insulin resistance likely is not valid since the disease in GK rats most probably had progressed from insulin resistance to overt diabetes.

Urinary TBARS $(\mu \mathrm{g} / 24 \mathrm{~h})$ was decreased with the higher level of antioxidant capacity whereas the content of $\beta$-glucan was positively associated with urinary TBARS excretion. Since TBARS is a less specific marker of lipid peroxidation, the role of whole grain intake on oxidative stress in GK rats was also determined by measuring the more specific marker, urinary 8 -isoprostane ${ }^{(43)}$. Using this marker, a significant interaction between antioxidant capacity and $\beta$-glucan content was found, such that the higher level of antioxidant activity decreased the excretion of urinary 8 -isoprostane only when the $\beta$-glucan content is low. Urinary TBARS and 8-isoprostane were positively correlated only at the 5 month time period $(r 0 \cdot 47, P<0 \cdot 009)$. A possible explanation of the lack of effect of the whole grains on oxidative stress would include poor bioavailability of the antioxidants from the whole grain. Whole grains, particularly within the bran fraction, are a rich source of phenolic compounds, which are potent antioxidants ${ }^{(44-46)}$. Since most phenolics from plant sources are bound to cell wall polymers, the physiological impact of grain phenolics depends on their bioavailability. It has been shown that the absorption of ferulic acid, a major phenolic compound in grain, is limited due to poor bioavailability of insoluble bound phenolics ${ }^{(47)}$.

It is well established that total $\beta$-cell mass in GK rats is decreased by more than $50 \%$ in the late fetal age and remains reduced into adulthood, relative to age-matched Wistar rats ${ }^{(30,48)}$. This $\beta$-cell deficit has been shown to result from impaired $\beta$-cell neogenesis ${ }^{(49)}$. The depletion of $\beta$-cells accelerates with age and with the consumption of a highsucrose diet, which induces severe hyperglycaemia and apoptosis of $\beta$-cells in GK rats ${ }^{(50)}$. Chronic exposure of $\beta$-cells to either hyperglycaemia or elevated concentrations of NEFA induces $\beta$-cell dysfunction and $\beta$-cell death, giving rise to the term 'glucolipotoxicity' ${ }^{(51,52)}$. In the present study, the total $\beta$-cell mass was not altered with the consumption of whole grains compared with the basal diet, which is consistent with the general lack of difference among diet groups in plasma glucose and NEFA concentrations at the end of the feeding period.

In the earliest stage of diabetic nephropathy (hypertrophyhyperfunction), glomerular filtration and kidney size are increased $^{(53)}$. Increased glomerular hyperfiltration has been shown to be correlated with kidney enlargement ${ }^{(54)}$. In GK rats, prolonged hyperglycaemia has been associated with increased kidney size at 6 months of age ${ }^{(55)}$, consistent with studies showing a strong relationship between the degree of kidney enlargement and the concentration of blood glu$\cos \mathrm{e}^{(56,57)}$. In the present study, relative kidney weight (g/100g body weight) was significantly decreased by the whole maize diet compared with the whole wheat and oats diets. We also found a statistically significant correlation between relative kidney weight and percentage of $\mathrm{GHb}$ measured at the end of the feeding trial. A significant main effect of antioxidant activity on both the relative kidney weight and GHb was found, such that greater antioxidant capacity was associated with a reduction in relative kidney weight and GHb. We have previously shown a reduction in kidney weight and plasma glucose concentration by antioxidant treatment in streptozotocin-induced diabetic rats ${ }^{(58)}$. Thus, grains with high antioxidant capacity may be beneficial in reducing kidney enlargement, an early stage in the development of diabetic nephropathy, relative to those with low antioxidant capacity.

In the present study, changes in renal hyperfiltration were evaluated by the measurement of CCr, which did not differ significantly among the diet groups at 5 months. However, the consumption of whole grains with a high level of $\beta$-glucan had slightly but significantly greater CCr compared with grains with low $\beta$-glucan, suggesting greater hyperfiltration with the consumption of $\beta$-glucan-rich grains. Given that reports of changes in $\mathrm{CCr}$ in GK rats, compared with agematched Wistar rats, are inconsistent, having been reported as elevated ${ }^{(59)}$ or unchanged ${ }^{(60)}$, it is uncertain whether $\beta$-glucan-rich grains may influence the development of diabetic nephropathy. The role of antioxidant activity and $\beta$-glucan from whole grains in altering the risk of diabetic renal disease warrants further investigation.

In conclusion, the consumption of whole grains overall did not demonstrate beneficial effects on glucose control, insulin resistance or on markers of oxidative stress in GK rats after 5 months of feeding. However, the finding of lower fasting plasma glucose in the wheat, barley and oats fed groups after 2 months of feeding raises the possibility of a modest beneficial effect early in the course of diabetes. Consumption of whole barley and oats might also be advantageous in slightly delaying the progression of type 2 diabetes by improving insulin resistance in the early stage of the disease. Cereals with high $\beta$-glucan content may also limit diabetes development at an early stage by improving short-term glucose control and insulin secretion. The consumption of whole grains with a high antioxidant capacity may also exert a beneficial effect on the development of type 2 diabetes through the reduction 
in relative kidney weight, GHb and oxidative stress. However, whether these effects result from high $\beta$-glucan content or antioxidant capacity or whether some other component of the whole grain is responsible for the above-mentioned effects will require further investigation.

\section{Acknowledgements}

The present study was supported by General Mills Bell Institute of Health and Nutrition (Minneapolis, MN, USA) and the Minnesota Agricultural Experimental Station. None of authors have any conflicts of interest. M. Y., A. S. C., and D. D. G. designed the research, M. Y. conducted the research, M. Y. and D. D. G. analysed the data and wrote the paper. D. D. G. had primary responsibility for the final content. We thank Cindy Gallaher for excellent technical assistance. We also thank Dr Robert L. Sorenson for the kind gift of the insulin antibody and for expert guidance on the quantification of $\beta$-cell mass. The authors gratefully acknowledge Dr Cyprian Weaver and John M. Basgen for providing excellent guidance on insulin immunohistochemistry and the point counting method, respectively.

\section{References}

1. Wild S, Roglic G, Green A, et al. (2004) Global prevalence of diabetes: estimates for the year 2000 and projections for 2030. Diabetes Care 27, 1047-1053.

2. US Department of Health and Human Services, Center for Disease Control and Prevention (2007) 2007 National Diabetes Fact Sheet. http://www.cdc.gov/diabetes/pubs/ estimates $07 . \mathrm{htm}$

3. van Dam RM (2003) The epidemiology of lifestyle and risk for type 2 diabetes. Eur J Epidemiol 18, 1115-1125.

4. Meyer KA, Kushi LH, Jacobs DR Jr, et al. (2000) Carbohydrates, dietary fiber, and incident type 2 diabetes in older women. Am J Clin Nutr 71, 921-930.

5. Fung TT, Hu FB, Pereira MA, et al. (2002) Whole-grain intake and the risk of type 2 diabetes: a prospective study in men. Am J Clin Nutr 76, 535-540.

6. Montonen J, Knekt P, Jarvinen R, et al. (2003) Whole-grain and fiber intake and the incidence of type 2 diabetes. $A m J$ Clin Nutr 77, 622-629.

7. Jang Y, Lee JH, Kim OY, et al. (2001) Consumption of whole grain and legume powder reduces insulin demand, lipid peroxidation, and plasma homocysteine concentrations in patients with coronary artery disease: randomized controlled clinical trial. Arterioscler Thromb Vasc Biol 21, 2065-2071.

8. Pereira MA, Jacobs DR Jr, Pins JJ, et al. (2002) Effect of whole grains on insulin sensitivity in overweight hyperinsulinemic adults. Am J Clin Nutr 75, 848-855.

9. Andersson A, Tengblad S, Karlstrom B, et al. (2007) Wholegrain foods do not affect insulin sensitivity or markers of lipid peroxidation and inflammation in healthy, moderately overweight subjects. J Nutr 137, 1401-1407.

10. Tighe P, Duthie G, Vaughan N, et al. (2010) Effect of increased consumption of whole-grain foods on blood pressure and other cardiovascular risk markers in healthy middle-aged persons: a randomized controlled trial. $\mathrm{Am} \mathrm{J}$ Clin Nutr 92, 733-740.
11. Hallfrisch J, Facn \& Behall KM (2000) Mechanisms of the effects of grains on insulin and glucose responses. $J \mathrm{Am}$ Coll Nutr 19, 320S-325S.

12. Pick ME, Hawrysh ZJ, Gee MI, et al. (1996) Oat bran concentrate bread products improve long-term control of diabetes: a pilot study. J Am Diet Assoc 96, 1254-1261.

13. Jenkins AL, Jenkins DJ, Zdravkovic U, et al. (2002) Depression of the glycemic index by high levels of beta-glucan fiber in two functional foods tested in type 2 diabetes. Eur J Clin Nutr 56, 622-628.

14. Torsdottir I, Alpsten M, Andersson H, et al. (1989) Dietary guar gum effects on postprandial blood glucose, insulin and hydroxyproline in humans. J Nutr 119, 1925-1931.

15. Leclere CJ, Champ M, Boillot J, et al. (1994) Role of viscous guar gums in lowering the glycemic response after a solid meal. Am J Clin Nutr 59, 914-921.

16. Rainbird AL, Low AG \& Zebrowska T (1984) Effect of guar gum on glucose and water absorption from isolated loops of jejunum in conscious growing pigs. Br J Nutr 52, 489-498.

17. Mayer-Davis EJ, Costacou T, King I, et al. (2002) Plasma and dietary vitamin $\mathrm{E}$ in relation to incidence of type 2 diabetes: The Insulin Resistance and Atherosclerosis Study (IRAS). Diabetes Care 25, 2172-2177.

18. Montonen J, Knekt P, Jarvinen R, et al. (2004) Dietary antioxidant intake and risk of type 2 diabetes. Diabetes Care 27, 362-366.

19. Halvorsen BL, Holte K, Myhrstad MC, et al. (2002) A systematic screening of total antioxidants in dietary plants. J Nutr 132, 461-471.

20. Bruce B, Spiller GA, Klevay LM, et al. (2000) A diet high in whole and unrefined foods favorably alters lipids, antioxidant defenses, and colon function. J Am Coll Nutr 19, 61-67.

21. Movassat J, Bailbe D, Lubrano-Berthelier C, et al. (2008) Follow-up of GK rats during prediabetes highlights increased insulin action and fat deposition despite low insulin secretion. Am J Physiol Endocrinol Metab 294, E168-E175.

22. Miller HE, Rigelhof F, Marquart L, et al. (2000) Antioxidant content of whole grain breakfast cereals, fruits and vegetables. J Am Coll Nutr 19, 312S-319S.

23. Goldstein DE, Little RR, Lorenz RA, et al. (2004) Tests of glycemia in diabetes. Diabetes Care 27, 1761-1773.

24. Mihara M \& Uchiyama M (1978) Determination of malonaldehyde precursor in tissues by thiobarbituric acid test. Anal Biochem 86, 271-278.

25. Lee HS, Shoeman DW \& Csallany AS (1992) Urinary response to in vivo lipid peroxidation induced by vitamin $\mathrm{E}$ deficiency. Lipids 27, 124-128.

26. Haffner SM, Kennedy E, Gonzalez C, et al. (1996) A prospective analysis of the HOMA model. The Mexico City Diabetes Study. Diabetes Care 19, 1138-1141.

27. Gundersen HJ, Bendtsen TF, Korbo L, et al. (1988) Some new, simple and efficient stereological methods and their use in pathological research and diagnosis. APMIS 96, 379-394.

28. Goto Y, Kakizaki M \& Masaki N (1975) Spontaneous diabetes produced by selective breeding of normal Wistar rats. Proc Jpn Acad 51, 80-85.

29. Hughes SJ, Suzuki K \& Goto Y (1994) The role of islet secretory function in the development of diabetes in the GK Wistar rat. Diabetologia 37, 863-870.

30. Movassat J, Saulnier C, Serradas P, et al. (1997) Impaired development of pancreatic beta-cell mass is a primary event during the progression to diabetes in the GK rat. Diabetologia 40, 916-925. 
31. Hussain MA (1997) Polygenic models of non-insulin-dependent diabetes mellitus. Eur J Endocrinol 137, 453-454.

32. Sachidanandam K, Elgebaly MM, Harris AK, et al. (2008) Effect of chronic and selective endothelin receptor antagonism on microvascular function in type 2 diabetes. Am J Physiol Heart Circ Physiol 294, H2743-H2749.

33. Tappy L, Gugolz E \& Wursch P (1996) Effects of breakfast cereals containing various amounts of beta-glucan fibers on plasma glucose and insulin responses in NIDDM subjects. Diabetes Care 19, 831-834.

34. Bunn HF, Gabbay KH \& Gallop PM (1978) The glycosylation of hemoglobin: relevance to diabetes mellitus. Science $\mathbf{2 0 0}$, $21-27$.

35. Lapolla A, Traldi P \& Fedele D (2005) Importance of measuring products of non-enzymatic glycation of proteins. Clin Biochem 38, 103-115.

36. Jain SK (1998) Glutathione and glucose-6-phosphate dehydrogenase deficiency can increase protein glycosylation. Free Radic Biol Med 24, 197-201.

37. Balamurugan R, Selvaraj N, Bobby Z, et al. (2007) Increased glycated hemoglobin level in non-diabetic nephrotic children is associated with oxidative stress. Indian J Physiol Pharmacol 51, 153-159.

38. Jain SK \& Palmer M (1997) The effect of oxygen radicals metabolites and vitamin E on glycosylation of proteins. Free Radic Biol Med 22, 593-596.

39. Horwitz DL, Starr JI, Mako ME, et al. (1975) Proinsulin, insulin, and C-peptide concentrations in human portal and peripheral blood. J Clin Invest 55, 1278-1283.

40. Kimura K, Toyota T, Kakizaki M, et al. (1982) Impaired insulin secretion in the spontaneous diabetes rats. Tohoku J Exp Med 137, 453-459.

41. Ohneda M, Johnson JH, Inman LR, et al. (1993) GLUT2 expression and function in beta-cells of GK rats with NIDDM. Dissociation between reductions in glucose transport and glucose-stimulated insulin secretion. Diabetes $\mathbf{4 2}$, 1065-1072.

42. Mari A, Ahren B \& Pacini G (2005) Assessment of insulin secretion in relation to insulin resistance. Curr Opin Clin Nutr Metab Care 8, 529-533.

43. Milne GL, Musiek ES \& Morrow JD (2005) F2-isoprostanes as markers of oxidative stress in vivo: an overview. Biomarkers 10, Suppl. 1, S10-S23.

44. Adom KK \& Liu RH (2002) Antioxidant activity of grains. J Agric Food Chem 50, 6182-6187.

45. Martinez-Tome M, Murcia MA, Frega N, et al. (2004) Evaluation of antioxidant capacity of cereal brans. J Agric Food Chem 52, 4690-4699.

46. Liyana-Pathirana CM \& Shahidi F (2006) Importance of insoluble-bound phenolics to antioxidant properties of wheat. J Agric Food Chem 54, 1256-1264.
47. Adam A, Crespy V, Levrat-Verny MA, et al. (2002) The bioavailability of ferulic acid is governed primarily by the food matrix rather than its metabolism in intestine and liver in rats. J Nutr 132, 1962-1968.

48. Miralles F \& Portha B (2001) Early development of beta-cells is impaired in the GK rat model of type 2 diabetes. Diabetes 50, Suppl. 1, S84-S88.

49. Movassat J, Calderari S, Fernandez E, et al. (2007) Type 2 diabetes - a matter of failing beta-cell neogenesis? Clues from the GK rat model. Diabetes Obes Metab 9, Suppl. 2, 187-195.

50. Koyama M, Wada R, Sakuraba H, et al. (1998) Accelerated loss of islet beta cells in sucrose-fed Goto-Kakizaki rats, a genetic model of non-insulin-dependent diabetes mellitus. Am J Pathol 153, 537-545.

51. Harmon JS, Gleason CE, Tanaka Y, et al. (2001) Antecedent hyperglycemia, not hyperlipidemia, is associated with increased islet triacylglycerol content and decreased insulin gene mRNA level in Zucker diabetic fatty rats. Diabetes $\mathbf{5 0}$ 2481-2486.

52. Prentki M, Joly E, El-Assaad W, et al. (2002) Malonyl-CoA signaling, lipid partitioning, and glucolipotoxicity: role in beta-cell adaptation and failure in the etiology of diabetes. Diabetes 51, Suppl. 3, S405-S413.

53. Mogensen CE \& Andersen MJ (1975) Increased kidney size and glomerular filtration rate in untreated juvenile diabetes: normalization by insulin-treatment. Diabetologia $\mathbf{1 1}$ 221-224.

54. Garcia Puig J, Mateos Anton F, Grande C, et al. (1981) Relation of kidney size to kidney function in early insulindependent diabetes. Diabetologia 21, 363-367.

55. Nobrega MA, Fleming S, Roman RJ, et al. (2004) Initial characterization of a rat model of diabetic nephropathy. Diabetes 53, 735-742.

56. Seyer-Hansen K (1977) Renal hypertrophy in experimental diabetes: relation to severity of diabetes. Diabetologia 13, $141-143$.

57. Gallaher DD, Olson JM \& Larntz K (1992) Dietary guar gum halts further renal enlargement in rats with established diabetes. J Nutr 122, 2391-2397.

58. Kim SS, Gallaher DD \& Csallany AS (2000) Vitamin E and probucol reduce urinary lipophilic aldehydes and renal enlargement in streptozotocin-induced diabetic rats. Lipids 35, 1225-1237.

59. Kim CS, Sohn EJ, Kim YS, et al. (2007) Effects of KIOM-79 on hyperglycemia and diabetic nephropathy in type 2 diabetic Goto-Kakizaki rats. J Ethnopharmacol 111, 240-247.

60. Sato N, Komatsu K \& Kurumatani H (2003) Late onset of diabetic nephropathy in spontaneously diabetic GK rats. Am J Nephrol 23, 334-342. 